\title{
COMPOSITION OF SONGS USING SYMMETRIC GROUP
}

\author{
${ }^{1}$ Adeniji, A. O., ${ }^{2}$ Eze, N. I and ${ }^{3}$ Mogbonju, M.M. \\ ${ }^{1,2,3}$ Department of Mathematics, Faculty of Science \\ University of Abuja, Abuja \\ 12adeniji4love@yahoo.com, ${ }^{2}$ mmogbonju@gmail.com and ${ }^{3}$ ezespalace1@gmail.com
}

\section{ABSTRACT}

Composition of songs involves the principle of key scaling, their respective interval as calculated with the aid of an appropriate key division which suites the generality of songs composed in different keys with the keyboard. Melodies, harmonies and rhythms produced in the stage of rendition is characterized by transposition and inversion of key to suite each song.

With the aid of keyboard, elements of Symmetric group are used to compose songs.

KEYWORDS: Symmetric group, diatonic scale, inversion of keys

Language: English

Date of Publication: 20-09-2018

DOI: 10.24297/jam.v14i2.7514

ISSN: $2347-1921$

Volume: 14 Issue: 02

Journal: Journal Of Advances In Mathematics

Website: https://cirworld.com

This work is licensed under a Creative Commons Attribution 4.0 International License. 


\section{INTRODUCTION}

\subsection{THE PYTHAGOREAN SCALE AND THE EQUAL TEMPERED SCALE}

Pythagorean scales are generated by a single pure interval. The simplest non-trivial harmonic ratios are $3 / 2$ (the perfect fifth) and $2 / 3$, which

normalizes (i.e., scaled by the appropriate power of two to lie in the "fundamental" octave of intervals between 1 and 2) to $4 / 3$ (the perfect fourth). As intervals, these ratios are closely related. Move up from the root by $3 / 2$ and then by $4 / 3$ and the note reached is $2 / 1$, or one octave. Starting with a root, moving up two perfect fifths produces a note that normalizes to $9 / 8$ from the root. This observation gives us one way to construct a sequence of notes from pure ratios. In general, the ratio of the nth note of this sequence from the root note has a simple formula involving log2(3). In this process, the normalized octave is noticeably sharp. Its normalized value is $\mathrm{s}$ approximately 1.0136. This ratio, now known as the Pythagorean Comma, presents a serious challenge for the Pythagorean system. Insisting that each new note be related by a perfect fifth to some previous note in our scale does not ensure that arbitrary pairs of notes have any kind of nice harmonic relationship. It also fails to ever generate a perfect octave.One historical solution to this problem was to temper the Pythagorean scale by flattening the twelfth ratio into a perfect octave as proposed by Steven [6]. In general, a temperament or tempered scale is any scale that results from adjusting the intervals of some other scale.

Most often, we hear twinkling sound emanating from a well composed music; the idea behind it is no other work than the all-powerful equal tempered scale. It is on this note that Laura [5] was prompted into exploring the world of the equal tempered scale having also explored the Pythagorean scale. He discovered some unique and interesting thing about the equal tempered scale - the diatonic scale of music composition that is, the scale is the $C$ to $C$ diatonic scale and chromatic scale.He explained that, the equal tempered scale came because of the de-consonance associated with the Pythagorean scale when it fails to meet up with the generality of the problem posed. As a result, musicians found it so very difficult to play and compose songs using the Pythagorean scale.

The difference between the seven octave and the twelve fifth of the Pythagorean scale was seen from the difference in its ratio and inequality in comparison. With this difference involved, it became difficult for musician to play music on this scale as one would not be able to play several keys of songs using this scale.

So, the need to create a scale which divides the interval of the 12-tone scale into twelve equal intervals arises. After a long research and hard work, a geometric progression approach was applied where $a=1$ and $r=\sqrt[12]{2}$ which divides the scale in twelve equal intervals thereby measuring the interval in cent; that $\sqrt[12]{2}$ is 100 cent par intervals. It was the perfect scale for music composition and highly adopted and used by musicians in playing song having different keys. This is how the tempered equal scale came about.

Timothy [8] presents the work of diatonic theorists who have focused on aspects of the diatonic collection over the past decades (the book is in fact dedicated to Clough [1,2], one of the most prominent figures in the field of diatonic set theory). It is intended as a supplemental text (not a replacement text) for a music fundamentals course, or as reading for a course on music and mathematics, or on diatonic set theory

\subsection{TRANSPOSITION AND INVERSION OF KEYS AND SCALES}

Another area where mathematics is very useful in music istransposition and inversion of keys using mathematics arithmetic modulo twelve (12) and seven (7) with the adoption of diatonic scale. It is in this regard that,

Thomas [7] made use of arithmetic modular in determining transposition and Inversion as functions $Z_{12} \rightarrow Z_{12}$ that are useful to every musician. There are also analogues for $Z_{7}$. Transposition and inversion are often applied to melodies, although they can also be applied to chords. Transposition mathematically captures what 
musicians do all the time: the restatement of a melody at higher and lower pitch levels in a way that preserves intervals. Inversion is another way to create musical variation while preserving the intervallic sound of a melody.

\subsection{PITCHES, RATIOS, SUBDIVISION OF SCALE AND OCTAVE DETERMINATION}

Interval determination between pitches, conversion of ratios into cents or semitones, subdivision of chromatic scale into twelve equal intervals and octave identification is one intrinsic aspect in music that mathematics is largely applied. David [4] discovered that the pitches $440 \mathrm{~Hz}\left(\mathrm{~A}_{4}\right)$ and $880 \mathrm{~Hz}$ differ, in that the latter has one octave above the former, hence $880 \mathrm{~Hz}$ is $A_{5}$. The pitch 220 is one octave below $A_{4}$, hence is $A_{3}$. The difference between the frequencies of $A_{3}$ and $A_{4}$ is 220 , while the difference between the frequencies of $A_{4}$ and $A_{5}$ is 440 , yet the intervals are the same - one octave, that is, not associated with the difference between two frequencies, but rather the ratio between the two frequencies.

He went further to say that to convert ratios into cents or semitones, logarithmic method of conversion is also appropriate. Meanwhile, as he investigated conversion of ratio, he also verged into the very possibilities of subdivision of chromatic scale into equal interval to suit a specific music composition. It is best that the subdivision be done as it resolves issues bordering on perfect music composition. For easy identification of notes, he brought forward the idea of numbering the chromatic scale which makes arithmetic modular twelve more appreciated as it is used extensively in composition of songs.

\subsection{DURATION OF NOTES AND SEQUENCE OF NOTES, DOTTED KEY NOTES AND DIVISION OF NOTES}

Time duration in music is one of the main constituents in music that without it, music would not be complete. To clearly bring to our understanding how time duration is important in music, David [4], analyzed how it associates to music by relating it with a beat produced also known as tempo in music. He explained saying that the representation of one beat in an interval is equivalent to a count-off time in measure per minute. He further explained that the basic designator of duration is the note and the duration of the note is determined by music signatures.

This gives rise to sequence of music signatures where each signature produces a sequence of their duration in a descending order. Each of these signatures is always seen in music composition and rendition.

His discovery also lead to his explaining how dotted note adds to the nitty-grittyof music. This is where he calculated the actual duration of a dotted note, bringing to our understanding the distinction between an undoted and dotted notes. Dotted notes have less value of time duration compared to the undotted notes. For more exquisite finishing in his work, he proposed that it is possible for note to be divided into several equal partition known as Tuplets.

\subsection{SYMMETRY IN MUSIC}

The word symmetry may be defined as a correct proportion of a part. In music, it is simply the even distribution of parts of melodies in rhyme. It is in this regard that Dave [3] explains the whole essence of symmetry in music as a symmetric group formulated in Mathematics.

\subsection{EQUAL TEMPERED SCALE}

Equal tempered scale is best achieved by creating a scale that divides the scale into equal parts giving it a twelve-tone equal scale.

An application of geometric progression method was adopted, where $\mathrm{a}=1$ and

$r=\sqrt[12]{2}$. 
This method is measured in 100 cents (the least measure that can be obtained for interval measurement on an instrument) per interval is derived as follows:

\begin{tabular}{|c|c|c|c|c|}
\hline C & $=$ & 1 & $=$ & 0 \\
\hline$C^{\#} / D^{b}$ & $=$ & $\sqrt[12]{2}$ & $=$ & 100 cents \\
\hline D & $=$ & $(\sqrt[12]{2})^{2}=$ & \multicolumn{2}{|c|}{200 cents } \\
\hline$D^{\#} / E^{b}$ & $=$ & $(\sqrt[12]{2})^{3}=$ & \multicolumn{2}{|c|}{300 cents } \\
\hline$E$ & $=$ & $(\sqrt[12]{2})^{4}=$ & \multicolumn{2}{|c|}{400 cents } \\
\hline $\mathrm{F}$ & $=$ & $(\sqrt[12]{2})^{5}=$ & \multicolumn{2}{|c|}{500 cents } \\
\hline$F^{\#} / G^{b}$ & $=$ & $(\sqrt[12]{2})^{6}=$ & \multicolumn{2}{|c|}{600 cents } \\
\hline G & $=$ & $(\sqrt[12]{2})^{7}=$ & \multicolumn{2}{|c|}{ 700cents } \\
\hline$G^{\#} / A^{b}$ & $=$ & $(\sqrt[12]{2})^{8}=$ & \multicolumn{2}{|c|}{800 cents } \\
\hline A & $=$ & $(\sqrt[12]{2})^{9}=$ & \multicolumn{2}{|c|}{900 cents } \\
\hline$A^{\#} / B^{b}$ & $=$ & $(\sqrt[12]{2})^{10}=$ & \multicolumn{2}{|c|}{1000 cents } \\
\hline B & $=$ & $(\sqrt[12]{2})^{11}=$ & \multicolumn{2}{|c|}{1100 cents } \\
\hline C & $=$ & 2 & $=$ & 1200 cents \\
\hline
\end{tabular}

This new formulated scale is the best scale used in playing several songs in different keys.

\section{INVERSION AND TRANSPOSITION OF KEYS AND SCALES}

Given a chromatic scale having keys ranging from 0 to 12 and carrying out addition on modulo 12 , we have.

$$
\begin{aligned}
& 1+2=3 \bmod 12 \\
& 6+5=11 \bmod 12 \\
& 11+1=0 \bmod 12
\end{aligned}
$$

Performing these on an addition modulo 12 table we have: 


\section{Table 1}

Representation of Chromatic Scale Addition modulo 12

\begin{tabular}{|l|llllllllllll|}
\hline+ & 0 & 1 & 2 & 3 & 4 & 5 & 6 & 7 & 8 & 9 & 10 & 11 \\
\hline 0 & 0 & 1 & 2 & 3 & 4 & 5 & 6 & 7 & 8 & 9 & 10 & 11 \\
2 & 1 & 2 & 3 & 4 & 5 & 6 & 7 & 8 & 9 & 10 & 11 & 0 \\
3 & 2 & 3 & 4 & 5 & 6 & 7 & 8 & 9 & 10 & 11 & 0 & 1 \\
4 & 3 & 4 & 5 & 6 & 7 & 8 & 9 & 10 & 11 & 0 & 1 & 2 \\
5 & 5 & 6 & 7 & 8 & 9 & 10 & 11 & 0 & 1 & 2 & 3 \\
6 & 6 & 7 & 8 & 9 & 10 & 11 & 0 & 1 & 2 & 3 & 4 & 5 \\
7 & 7 & 8 & 9 & 10 & 11 & 0 & 1 & 2 & 3 & 4 & 5 & 6 \\
8 & 8 & 9 & 10 & 11 & 0 & 1 & 2 & 3 & 4 & 5 & 6 & 7 \\
9 & 9 & 10 & 11 & 0 & 1 & 2 & 3 & 4 & 5 & 6 & 7 & 8 \\
10 & 10 & 11 & 0 & 1 & 2 & 3 & 4 & 5 & 6 & 7 & 8 & 9 \\
11 & 11 & 0 & 1 & 2 & 3 & 4 & 5 & 6 & 7 & 8 & 9 & 10 \\
\hline
\end{tabular}

Let $C=0, C^{\#} / D^{b}=1, D=2, D^{\#} / E^{b}=3, E=4, F=5, F^{\#} / G^{b}=6, G=7$,

$G^{\#} / A^{b}=8, A=9, A^{\#} / B^{b}=10, B=11$.

Substituting into table 1 appropriately yields:

Table 2

Keys Representing Elements of Addition Modulo 12

\begin{tabular}{|l|llllllllllll|}
\hline+ & $\mathrm{C}$ & $\mathrm{C}^{\#}$ & $\mathrm{D}$ & $\mathrm{D}^{\#}$ & $\mathrm{E}$ & $\mathrm{F}$ & $\mathrm{F}^{\#}$ & $\mathrm{G}$ & $\mathrm{G}^{\#}$ & $\mathrm{~A}$ & $\mathrm{~A}^{\#}$ & $\mathrm{~B}$ \\
\hline $\mathrm{C}$ & $\mathrm{C}$ & $\mathrm{C}^{\#}$ & $\mathrm{D}$ & $\mathrm{D}^{\#}$ & $\mathrm{E}$ & $\mathrm{F}$ & $\mathrm{F}^{\#}$ & $\mathrm{G}$ & $\mathrm{G}^{\#}$ & $\mathrm{~A}$ & $\mathrm{~A}^{\#}$ & $\mathrm{~B}$ \\
$\mathrm{C}^{\#}$ & $\mathrm{C}^{\#}$ & $\mathrm{D}$ & $\mathrm{D}^{\#}$ & $\mathrm{E}$ & $\mathrm{F}$ & $\mathrm{F}^{\#}$ & $\mathrm{G}$ & $\mathrm{G}^{\#}$ & $\mathrm{~A}$ & $\mathrm{~A}^{\#}$ & $\mathrm{~B}$ & $\mathrm{C}$ \\
$\mathrm{D}$ & $\mathrm{D}$ & $\mathrm{D}^{\#}$ & $\mathrm{E}$ & $\mathrm{F}$ & $\mathrm{F}^{\#}$ & $\mathrm{G}$ & $\mathrm{G}^{\#}$ & $\mathrm{~A}$ & $\mathrm{~A}^{\#}$ & $\mathrm{~B}$ & $\mathrm{C}$ & $\mathrm{C}^{\#}$ \\
$\mathrm{D}^{\#}$ & $\mathrm{D}^{\#}$ & $\mathrm{E}$ & $\mathrm{F}$ & $\mathrm{F}^{\#}$ & $\mathrm{G}$ & $\mathrm{G}^{\#}$ & $\mathrm{~A}$ & $\mathrm{~A}^{\#}$ & $\mathrm{~B}$ & $\mathrm{C}$ & $\mathrm{C}^{\#}$ & $\mathrm{D}$ \\
$\mathrm{E}$ & $\mathrm{E}$ & $\mathrm{F}$ & $\mathrm{F}^{\#}$ & $\mathrm{G}$ & $\mathrm{G}^{\#}$ & $\mathrm{~A}$ & $\mathrm{~A}^{\#}$ & $\mathrm{~B}$ & $\mathrm{C}$ & $\mathrm{C}^{\#}$ & $\mathrm{D}$ & $\mathrm{F}$ \\
\hline
\end{tabular}




\begin{tabular}{l|llllllllllll}
$F$ & $F$ & $F^{\#}$ & $G$ & $G^{\#}$ & $A$ & $A^{\#}$ & $B$ & $C$ & $C^{\#}$ & $D$ & $F$ & $F^{\#}$ \\
$F^{\#}$ & $F^{\#}$ & $G$ & $G^{\#}$ & $A$ & $A^{\#}$ & $B$ & $C$ & $C^{\#}$ & $D$ & $F$ & $F^{\#}$ & $G$ \\
$G$ & $G$ & $G^{\#}$ & $A$ & $A^{\#}$ & $B$ & $C$ & $C^{\#}$ & $D$ & $F$ & $F^{\#}$ & $G$ & $G^{\#}$ \\
$G^{\#}$ & $G^{\#}$ & $A$ & $A^{\#}$ & $B$ & $C$ & $C^{\#}$ & $D$ & $F$ & $F^{\#}$ & $G$ & $G^{\#}$ & $A$ \\
$A$ & $A$ & $A^{\#}$ & $B$ & $C$ & $C^{\#}$ & $D$ & $F$ & $F^{\#}$ & $G$ & $G^{\#}$ & $A$ & $A^{\#}$ \\
$A^{\#}$ & $A^{\#}$ & $B$ & $C$ & $C^{\#}$ & $D$ & $F$ & $F^{\#}$ & $G$ & $G^{\#}$ & $A$ & $A^{\#}$ & $B$ \\
$B$ & $B$ & $C$ & $C^{\#}$ & $D$ & $F$ & $F^{\#}$ & $G$ & $G^{\#}$ & $A$ & $A^{\#}$ & $B$ & $C$
\end{tabular}

\section{Definition 2.1}

Let $\mathrm{n}$ be an integer mod twelve. Then the function $T_{n}: Z_{12} \rightarrow Z_{12}$ defined by the formula $T_{n}(x)=x+$ $n \bmod 12$ is called transposition about $n$.

\section{Example 2.1}

Given $\left.T_{7}: Z_{12} Z_{12}-x\right)=x+\bmod 12$

\section{Solution:}

$$
\begin{aligned}
& T_{7}(3)=3+7=10 \\
& T_{7}(6)=6+7=1 \\
& T_{7}(7)=7+7=2 \\
& T_{7}(10)=10+7=5
\end{aligned}
$$

\section{Definition 2.2}

Let $n$ be an integer mod twelve. Then the function $T_{n}: Z_{12} Z_{12}$ defined by $\longrightarrow$

The formula $T_{n}(x)=-x+n \bmod 12$ is called inversion about $\mathrm{n}$.

\section{Example 2.2}

Given $T_{7}: Z_{12} Z_{12}$ find $P_{n}(x)=-x+n \bmod 12$

\section{Solution}

$$
\begin{aligned}
& T_{7}(3)=-3+7=4 \\
& T_{7}(7)=-7+7=0 \\
& T_{7}(9)=-9+7=2 \\
& T_{7}(10)=-10+7=3
\end{aligned}
$$




\section{PITCH CLASS RATIO AND RATIO CONVERSION}

Each note on the keyboard represents a pitch having various frequencies.

Given that key of $\quad A_{4}=440 h z$ and $A_{5}=880 h z$

The note or key $A_{4}$ and $A_{5}$ are different pitches whose frequencies are one octave higher than the other.

That is $A_{5}>\quad A_{4}$.

The octave frequency difference is 2 times the interval of its frequencies which is best associated with the ratio of $A_{4}$ and $A_{5}$ which may be denoted asf $f_{1}$ and $f_{2}\left(f_{1}, f_{2}\right)$. The pair is of equivalent class known as the associated ratio in terms of intervals. The ratio of $f_{1}: f_{2}$ given as

$r=\frac{f_{1}}{f_{2}}$ is the measurement of the interval between the pitches and is called interval ratio.

$f_{2}>f_{1}$ is upward or monotonic increasing

$f_{2}<f_{1}$ is downward or monotonic decreasing

\section{Definition 3.1}

Measurement of interval by ratio among pitches is called multiplicative, while measurement by interval in semitone, steps or octaves are called additive.

\section{CONVERSION OF SEMITONE TO RATIO AND CENTS TO RATIO.}

Let $a$ be a real number and since twelve iteration of an interval gives an octave which has the ratio 2 , we have.

$$
a^{12}=2
$$

$a=\sqrt[12]{2}=2^{\frac{1}{12}}$ thus, for $n$ semitone, the ratio equals

$$
\left(2^{1 / 12}\right)^{n}=2^{n / 12}
$$

Let $b$ be the ratio corresponding to a cent and let 1200 iteration equals an octave, then

$b^{1200}=2$ implies $\quad b=2^{1 / 1200}$

Hence, any $x$ cent $=2^{x / 1200}$ ratio

\section{CONVERSION OF RATIO TO CENT AND SEMITONE}

Let $x$ and $y$ be frequencies $f_{1}$ and $f_{2}$ and are an octave apart. Applying logarithmic method, we have: Let $\frac{x}{y}=2$

$\log _{b}(x / y)=\log _{b} 2 \quad$ implies $\log _{b} x-\log _{b} y=\log _{b} 2$

Let

$\log _{b} x-\log _{b} y=1$, so that

$$
\log _{b} 2=1 \Rightarrow b^{1}=2 \Rightarrow b=2
$$


The desired base.

Given $r$ ratio of interval, to convert in cent, from (4.1);

$r=2^{x / 1200}$

Multiplying both sides of (5.1) by $\log _{2}$, we have,

$$
\log _{2} r=\log _{2}\left(2^{x / 1200}\right) \text { implies } \log _{2} r=x / 1200
$$

$x=1200 \log _{2} r$

So, for any interval, ratio ' $r$ ' is measured in cents.

\section{CONVERSION OF RATIO TO SEMITONE}

Let $r=2^{n / 12} n=1,2,3 \ldots$

Where $r$, is the geometric progression which subdivides the chromatic scale to obtain an evenly equal division of the scale, the desired scale.

Multiplying both sides by $\log _{2}$, (6.1) becomes.

$$
\log _{2} r=\log _{2}\left(2^{n / 12}\right)=n / 12
$$

$r=12 \log _{2} r$

Hence for any interval $x$, the ratio is measured in semitone as (6.1).

\section{DURATION OF NOTES AND SEQUENCE OF NOTES}

Times duration in music are often measured in beats, which are the temporal units by which music is notated. Frequently, one beat represents the time interval by which one would "count off"; the passing of time while the music is performed. The term tempo refers to the frequency of this count off usually measured in beats per minute. In a musical score, the basic designator of duration is, of cause, the note. The duration of notes is determined by such things as note heads, stem flag dots, ties and tuplet designations. The duration in beats (often four) dictated by the time signature. Notes are assigned a sequence of beats in $1 / 2^{n}$ where $n \geq 0$. It is also denoted by a symbol having different forms like; semibreve or full note, mini or half note, crotchet or quarter note, quaver or one-eight note, semiquaver or one-sixteenth note, semihemiquaver or one-thirty-two note and semihemidemiquaver or one-sixty-four note. All in the sequence of numbers as shown below:
$\left\{1 / 2^{\mathrm{n}}\right\}$
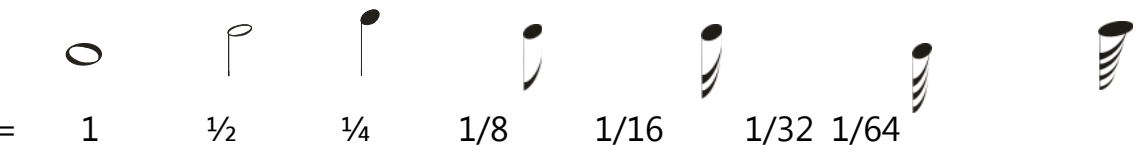

It represents the duration as it appears in music. As we move from left to right in the sequence, the duration of the sound produce shortens up to the sixty-fourth note as the least note.

In adjacent notes, flags may be replaced by beams connecting the stems:
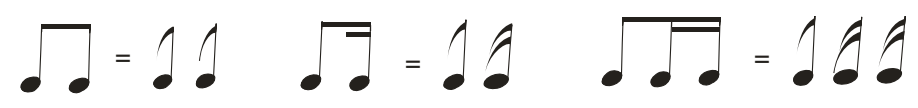


\section{DURATION OF A DOTTED KEY SIGNATURE}

A dot in front of a note or a rest adds to it one half of its original duration.

Assume a quarter duration note in four beats with dot in front of it, then

$$
\frac{1}{4}\left(1+\frac{1}{2}\right)=\frac{1}{4} \cdot \frac{3}{2}=\frac{3}{8}
$$

Where $1 / 4$ is the duration of the sixteenth note in beat multiplied by the duration of the dot in front i.e. $1+1 / 2$ which then yields $3 / 8$ duration of the sixteenth note in beat.In general terms in calculating the duration of any note with dot in front, we have as in [4]: Let $d$ be duration of note followed by $m$ has duration $d_{m}$ given by:

$$
\begin{gathered}
d_{m}=d\left(1+\frac{1}{2}+\frac{1}{2^{2}}+, \ldots,+\frac{1}{2^{n}}\right) \\
d_{m}=d \sum_{i=0}^{m}\left(\frac{1}{2}\right)^{i} \\
=d\left[\frac{1-\left(\frac{1}{2}\right)^{m+i}}{1-\frac{1}{2}}\right] \\
=d\left[\frac{1-\left(\frac{1}{2}\right)^{m+i}}{1-\frac{1}{2}}\right] \\
=d\left[2\left(1-\left(\frac{1}{2}\right)^{m+i}\right)\right] a s i=0 \\
=d\left[2\left(1-\left(\frac{1}{2}\right)^{m}\right)\right] \\
=d\left[2-\left(\frac{1}{2}\right)^{m}\right] \\
=d\left[1+\frac{2^{m}-1}{2^{m}}\right] \\
=d\left[1+1-\frac{1}{2^{m}}\right]
\end{gathered}
$$

Where $\frac{1-\left(\frac{1}{2}\right)^{m+1}}{1-\frac{1}{2}}$ is $\sum_{i=0}^{m} r^{i}=1+r+r^{2}+\cdots r^{n}=\frac{1-r^{m+1}}{1-r}$

which holds for any integer $m \geq 0$ and any real number $r \neq 1$.

A note of duration $d$ followed by $m$ dots has duration

$$
d_{m}=d\left[2-\left(\frac{1}{2}\right)^{m}\right]
$$


By convergence of sequences,

$\lim _{m \rightarrow \infty}\left[2-\left(\frac{1}{2}\right)^{m}\right]=2$

Hence, by mathematical induction

$\lim _{\substack{m \rightarrow \infty \\ i \geq 0}}\left[2-\left(\frac{1}{2}\right)^{m+i}\right]=2$

\section{Example 8.1}

What is the duration of a triply dotted thirty-second note?

\section{Solution}

Thirty-second note is $1 / 32$ and from the question it is dotted by a triply duration meaning $m$ is 3 . First, we must calculate the duration thirty-second note before being dotted and is $2 d$ which arises from the limit.

Hence

$$
d=\frac{1}{32} \cdot 2=\frac{1}{16}
$$

The number of dots $(m)$ is 3, we have,

$$
d_{3}=\frac{1}{16}\left[2-\left(\frac{1}{2}\right)^{3}\right]=\frac{1}{16}\left[2-\frac{1}{8}\right]=\frac{1}{16} \cdot \frac{15}{8}=\frac{15}{128}
$$

The duration is $\frac{15}{128}$ th beat. In general, the numerator of the duration of any triply dotted note is always 15 . In section 11 of this work, $d_{m}$ can be known with the formula stated.

\section{DIVISION OF NOTE}

A $\frac{1}{2^{n}}$ th note can be subdivided into $k$ equal note, where $k$ is not a power of 2 . The division of note into $k$ equal note is what is called a Tuplet where $k$ forms a $k$-tuplet.Given

$$
2^{r}<k<2^{r+1}
$$

Where $r$ is a positive integer, the $k$-tupletof a note is given as

$$
\frac{1}{2^{n+1}} \text { th note. }
$$

\section{Example 2}

For 3-tuplet note we have

$$
3 \cdot \frac{1}{2^{n+1}}=3 \cdot \frac{1}{2^{2+1}}=3 \cdot \frac{1}{2^{3}}=3 \cdot \frac{1}{8}=\frac{3}{8}
$$

This forms an eight not overset by 3 , forming an eighth note triplet.

Represented in a treble clef below: 


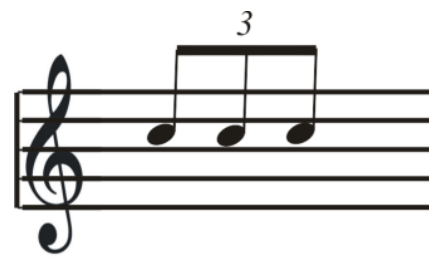

\section{Example 9.1}

What is the division of a quarter note into five equal notes?

\section{Solution}

Same method is applied as in example 2, but a slight difference.
A quarter note is

$$
=\frac{1}{4} \text { th note }=\quad \frac{1}{2^{2}} \text { th note }
$$

Given,

$2^{r}<k<2^{r+1}$

Since we are requested to divide the quarter note into 3 equal pieces, we have

$2^{2}<5<2^{3}=2^{1}<5<2^{2}$

Where $r=2$ and $k=5$

For 5-tuplet note we have

$$
5 \cdot \frac{1}{2^{n+1}}=5 \cdot \frac{1}{2^{2+2}}=5 \cdot \frac{1}{2^{4}}=5 \cdot \frac{1}{16}=\frac{5}{16}
$$

This forms a sixteenth not overset by 5, forming a sixteenth note triplet.

Represented in a treble clef below;

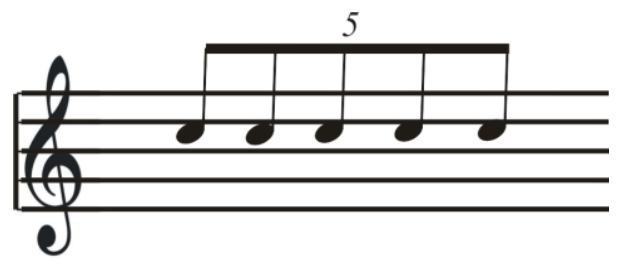

\section{SYMMETRIC GROUP}

Permutation can also be multiplied giving rise to certification of associative property and revealing identity and inverse element too. Let $X$ be a set such that $a$ and $b$ are its subsets.

Let

$$
a=\left(\begin{array}{lllll}
1 & 2 & 3 & 4 & 5 \\
2 & 5 & 1 & 4 & 3
\end{array}\right)=\left(\begin{array}{llll}
1 & 2 & 5 & 3
\end{array}\right)(4)
$$




$$
\begin{gathered}
b=\left(\begin{array}{lllll}
1 & 2 & 3 & 4 & 5 \\
3 & 5 & 4 & 1 & 2
\end{array}\right)=(134)\left(\begin{array}{lll}
2 & 5
\end{array}\right) \\
a b=\left(\begin{array}{lllll}
1 & 2 & 3 & 4 & 5 \\
5 & 2 & 3 & 1 & 4
\end{array}\right)=\left(\begin{array}{lll}
1 & 5 & 4
\end{array}\right) \text { and } b a=\left(\begin{array}{lll}
2 & 3 & 4
\end{array}\right) .
\end{gathered}
$$

Given $S_{4}=4 !=24$ elements as follows:

$$
\begin{aligned}
& \left(\begin{array}{lll}
1 & 23 & 4 \\
1 & 23 & 4
\end{array}\right),\left(\begin{array}{lll}
1 & 23 & 4 \\
1 & 24 & 3
\end{array}\right),\left(\begin{array}{lll}
1 & 23 & 4 \\
1 & 32 & 4
\end{array}\right),\left(\begin{array}{lll}
1 & 23 & 4 \\
1 & 34 & 2
\end{array}\right) \\
& \left(\begin{array}{lll}
1 & 23 & 4 \\
1 & 42 & 3
\end{array}\right),\left(\begin{array}{lll}
1 & 23 & 4 \\
1 & 43 & 2
\end{array}\right),\left(\begin{array}{lll}
1 & 23 & 4 \\
2 & 13 & 4
\end{array}\right),\left(\begin{array}{lll}
1 & 23 & 4 \\
2 & 14 & 3
\end{array}\right) \\
& \left(\begin{array}{lll}
1 & 23 & 4 \\
2 & 31 & 4
\end{array}\right),\left(\begin{array}{lll}
1 & 23 & 4 \\
2 & 34 & 1
\end{array}\right),\left(\begin{array}{lll}
1 & 23 & 4 \\
2 & 41 & 3
\end{array}\right),\left(\begin{array}{lll}
1 & 23 & 4 \\
2 & 34 & 1
\end{array}\right) \\
& \left(\begin{array}{lll}
1 & 23 & 4 \\
3 & 12 & 4
\end{array}\right),\left(\begin{array}{lll}
1 & 23 & 4 \\
3 & 14 & 2
\end{array}\right),\left(\begin{array}{lll}
1 & 23 & 4 \\
3 & 21 & 4
\end{array}\right),\left(\begin{array}{lll}
1 & 23 & 4 \\
3 & 24 & 1
\end{array}\right) \\
& \left(\begin{array}{lll}
1 & 23 & 4 \\
3 & 41 & 2
\end{array}\right),\left(\begin{array}{lll}
1 & 23 & 4 \\
3 & 42 & 1
\end{array}\right),\left(\begin{array}{lll}
1 & 23 & 4 \\
4 & 12 & 3
\end{array}\right),\left(\begin{array}{lll}
1 & 23 & 4 \\
4 & 13 & 2
\end{array}\right) \\
& \left(\begin{array}{lll}
1 & 23 & 4 \\
4 & 21 & 3
\end{array}\right),\left(\begin{array}{lll}
1 & 23 & 4 \\
4 & 23 & 1
\end{array}\right),\left(\begin{array}{lll}
1 & 23 & 4 \\
4 & 31 & 2
\end{array}\right),\left(\begin{array}{lll}
1 & 23 & 4 \\
4 & 32 & 1
\end{array}\right)
\end{aligned}
$$

\subsection{TRANSPOSITION AND INVERSION OF SONGS}

Transposition and inversion play a major role in diversifying music to a large extent. Looking at the chromatic scale of major triads, we shall see that successive keys of notes are derived by transposing and inverting one key to another.

\subsection{DIATONIC SCALE OF KEY OF C}

$C=\{C, D, E, F, G, A, B\}=\{d, r, m, f, s, l, t\}$

On the chromatic scale, it is represented as

$\begin{array}{llllllllllll}\mathrm{C} & \mathrm{C}^{\#} & \mathrm{D} & \mathrm{E}^{\mathrm{b}} & \mathrm{E} & \mathrm{F} & \mathrm{F}^{\#} & \mathrm{G} & \mathrm{A}^{\mathrm{b}} & \mathrm{A} & \mathrm{B}^{\mathrm{b}} & \mathrm{B} \\ 0 & 1 & 2 & 3 & 4 & 5 & 6 & 7 & 8 & 9 & 10 & 11\end{array}$

The sol-fa chords of key of $C$ are;

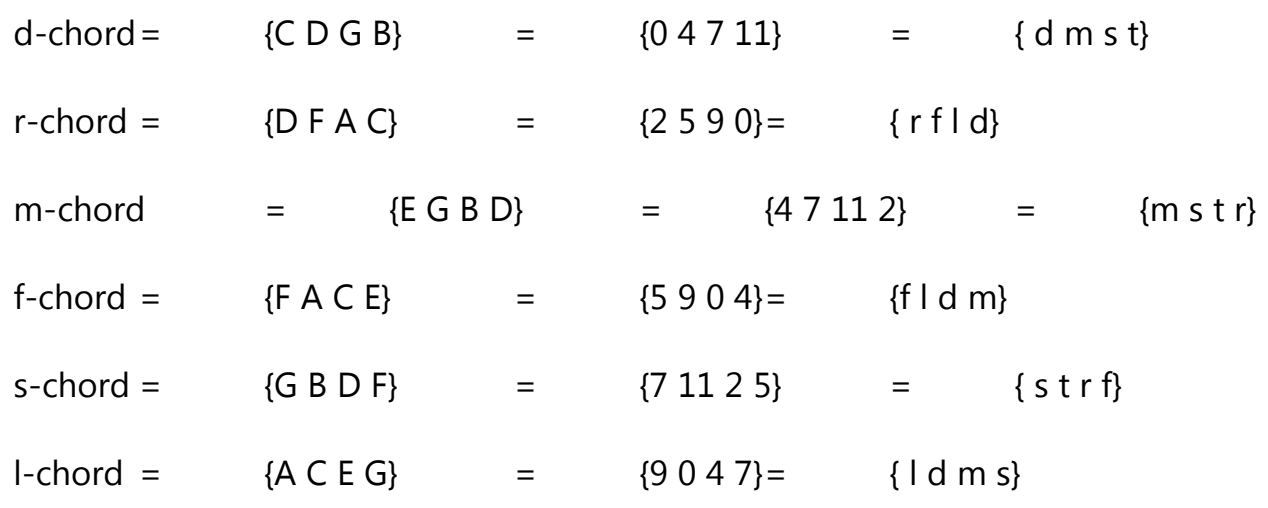


$\mathrm{t}$-chord $=\{$ BDFA $\quad=\{11259\}=\{\mathrm{trfl}\}$

This is the sol-fa chord of key of C. It can be transposed to another key applying the formula of transposition given as:

$T_{n}(x)=x+n$ 10.1The answer obtained as it is

known, is in arithmetic modulo twelve (12).

\subsection{TRANSPOSING KEY OF C TO G}

Diatonic Scale of key $G=\left\{G A B C D E F^{\#}\right\}=\{d r m f s \mid t\}$

Since the count between key of $C$ and key of $G$ is 7 , the transposition is doneby substituting each value of solfa chord of key of $C$ into equation 10.1 to have:

$T_{7}(0)=0+7=7, \quad T_{7}(4)=4+7=11, \quad T_{7}(7)=7+7=2$

$T_{7}(11)=11+7=6$. This yields the d-chord of $\mathrm{G}$ :

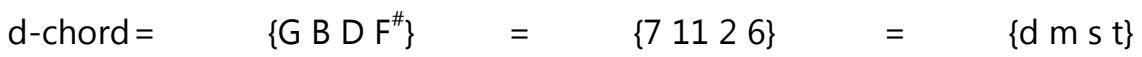

Carrying out the same calculation on $r, m, f, s, l$, t-chords of key of $C$ and by substituting appropriately yields the remaining solfa chords of $\mathrm{G}$ as shown below:

\begin{tabular}{|c|c|c|c|c|c|}
\hline r-chord = & $\{A \subset E G\}$ & $=$ & $\left\{\begin{array}{llll}9 & 0 & 4 & 7\end{array}\right\}=$ & \multicolumn{2}{|l|}{$r f \mid d$} \\
\hline m-chord & \multicolumn{2}{|c|}{$B D F^{\#} A$} & $=$ & $\{11269\}$ & $\{\mathrm{mstr}\}$ \\
\hline f-chord $=$ & $\{C E G B\}$ & $=$ & $\left\{\begin{array}{llll}0 & 9 & 71\end{array}\right\}$ & $=$ & $\{f \mid d m\}$ \\
\hline s-chord = & $\left\{D F^{\#} A C\right\}$ & $=$ & $\left\{\begin{array}{lll}2 & 6 & 9\end{array}\right\}=$ & $\{s t r f\}$ & \\
\hline |-chord = & $\{E G B D\}$ & $=$ & $\{47112\}$ & $=$ & $\{\mid \mathrm{d} m \mathrm{~s}\}$ \\
\hline t-chord $=$ & $\left\{F^{\#} A \subset E\right\}$ & $=$ & $\left\{\begin{array}{llll}6 & 9 & 0 & 4\end{array}\right\}=$ & $\{t r f l\}$ & \\
\hline
\end{tabular}

\subsection{TRANSPOSING KEY OF C TO KEY OF C}

Diatonic Scale of $C^{\#}=\left\{C^{\#} E^{b} F F^{\#} A^{b} B C\right\}=\{d r m f s \mid t\}$. Since the count between key of $C$ to key of $C^{\#}$ is 1 in the chromatic scale, transposition of key of $C^{\#}$ is also obtained in a similar way as of key of $G$ above, giving rise to the sol-fa chords of $C^{\#}$ as follows:

$$
T_{1}(0)=0+1=1, \quad T_{1}(4)=4+1=5, \quad T_{1}(7)=7+1=8
$$

$T_{1}(11)=11+1=0$

\begin{tabular}{|c|c|c|c|c|c|c|}
\hline d-chord = & $\left\{C^{\#} F A^{b} C\right\}$ & & $\multicolumn{2}{|c|}{\begin{array}{llll}1 & 5 & 8 & 0\end{array}}=$ & \multicolumn{2}{|l|}{$d m s t$} \\
\hline r-chord = & $\left\{E F B^{b} C^{\#}\right\}$ & & $=$ & $\left\{\begin{array}{llll}3 & 6 & 10 & 1\end{array}\right\}$ & $=$ & $\{r f \mid d\}$ \\
\hline m-chord & $\{F A$ & & $=$ & $\multicolumn{2}{|l|}{\begin{array}{llll}5 & 8 & 0 & 3\end{array}}=$ & $\{\mathrm{mstr}\}$ \\
\hline f-chord $=$ & $\left\{F^{\#} B^{b} C^{\#} F\right\}$ & $=$ & $\left\{\begin{array}{llll}6 & 10 & 1 & 5\end{array}\right\}$ & $=$ & $\{f \mid d m\}$ & \\
\hline s-chord = & $\left\{A^{b} C E^{b} F^{\#}\right\}$ & $=$ & $\{8036\}=$ & $\{s \operatorname{trf}\}$ & & \\
\hline
\end{tabular}


I-chord $=\left\{B^{b} C^{\#} F A^{b}\right\}=\left\{\begin{array}{lllll}10 & 1 & 5 & 8\end{array}\right\}=\{l d m s\}$

$\mathrm{t}$-chord $=\left\{C E^{b} F^{\#} B^{b}\right\}=\left\{\begin{array}{llll}0 & 3 & 6 & 10\end{array}\right\}=\{\mathrm{trf} \mid\}$

The diatonic scale of $C$ in the chromatic scale is most used here because it is the neutral key of the chromatic scale. It also implies that, key of $\mathrm{C}$ can also be transposed to any diatonic scale of any key in the chromatic scale vis-à-vis

\subsection{FUGUE OF D MINOR IN THE WELL-TEMPERED CLAVIER}

\section{BOOK 1}

Fugue music is one of the most classical western music compositions that mostly constitute transposition and inversion. Here we shall analyze Johann Sebastian Bach (1685-1750) fugue music composition of the welltempered Clavier Book 1, to show how transposition and inversion makes up classical piece of Bach composition.

FUGA VI.
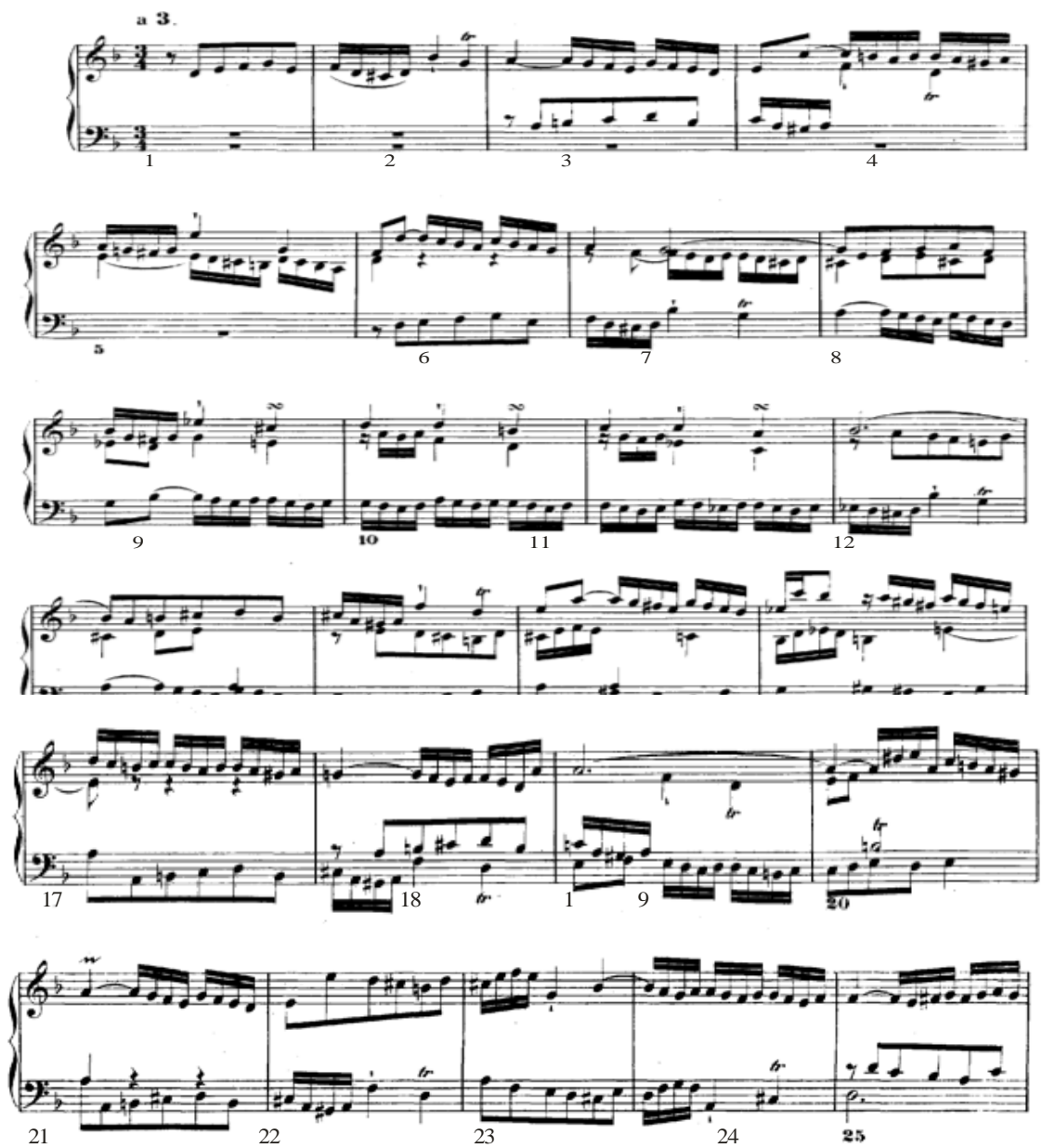

The number beneath each stave indicates various measures of the song.

The subject in measure 1 (Treble clef) is read as:

$\left\{D, E, F, G, E, F, D, C^{\#}, D, B^{b} ; G, A\right\}=\{2,4,5,7,4,5,2,1,2,10,7,9\}$

It lasts until the beginning of measure 3. Let it be $P$. 
Looking at the subject $\mathrm{P}$, we observed that it consists of twelve notes running into

measure 3. In measure 3 of the same stave (Bass clef) another melody goes out.

$\left\{A, B, C, D, B, C, A, G^{\#}, A, F, D, E\right\}=\{9,11,0,2,11,0,9,8,9,5,2,4\}$

In measure 6 on the (Bass clef) which runs till beginning of measure 8 , the subject returns in the exact same form as the introduction; just one octave lower. At measure 8 (Treble clef), a form of the subject run from 8 to the beginning of measure 10 and read as:

$\left\{E, F, G, A, F, B^{b} G, F^{\#}, G, E^{b}, C^{\#}, D\right\}=\{4,5,7,9,5,10,7,6,7,3,1,2\}$

This one does not entirely match though. The first five pitches

are almost transposition of the first five pitches of $P$, but the next 5 pitches are transposition of the respective pitches of $P$. The last pitch is also transposition of the $P$, but the eleventh pitch doesn't match. At measure 13 (Treble clef) running into the beginning of 16 , we have:

$\left\{A, B, C^{\#}, D, B, C^{\#}, A, C^{\#}, A, G^{\#}, F, D, E\right\}=\{9,11,1,2,11,1,9,8,9,5,2,4\}$

This is likethe transposition in key of $\mathrm{G}$ as in measure 3, except for the highlighted 1's. Measures 17,18, and 21 are respectively

$\left\{A, B, C, D, B, C^{\#}, A, G^{\#}, A, F, D, E\right\}=\{9,11,0,2,11,1,9,8,9,5,2,4\}$

$\left\{A, B, C^{\#}, D, B, C, A, G^{\#}, A, F, D, E\right\}=\{9,11,1,2,11,0,9,8,9,5,2,4\}$

$\left\{A, B, C^{\#}, D, B, C^{\#}, A, G^{\#}, A, F, D, E\right\}=\{9,11,1,2,11,1,9,8,9,5,2,4\}$

These are also transposition in key of $\mathrm{G}$ except for the highlighted 1's. The interval 7 is very important in western music and is called the perfect fifth. Here we see that transposition by a perfect fifth occurs four times before the piece is even half over. In fact, many fugues have this property. So far we have seen that transposition plays a role in this piece.

Looking at the inversion part of the fugue, consider measures 14 (Bass clef) running into the beginning of measure16 and measure 22 (Treble Clef) running into the beginning of measure 24 . They are respectively:

$\left\{E, D, C^{\#}, B, D, C, E, F, E, A, C, B^{b}\right\}=\{4,2,1,11,2,1,4,5,4,9,0,10\}$

$\left\{E, D, C^{\#}, B, D, C^{\#}, E, F, E, G, B^{b}, A\right\}=\{4,2,1,11,2,1,4,5,4,7,10,9\}$

They are nearly identical, except for the last three digits. Notice also that the first

two elements $\mathrm{E}$, Dare the same first two elements of $P$, just the order is switched. The last three notes of 22 are even the last three notes of $P$, just the order is switched. Calculating inversion 6 gives

$$
\{4,2,1,11,2,1,4,5,4,8,11,9\}
$$

which is a near perfect fifth with measures 14 and 22! Just the last three notes are changed to make it sound better. So, we see that inversion does indeed play a rolein the piece. 


\section{SONG TRANSPOSITION “EVERY VALLEY SHALL BE EXALTED - BY HANDEL GEORGE FRIDERICK (HANDEL MESSIAH) FROM KEY OF A TO KEY OF B AND KEY OF C.}

Analyzing this song to show its transposition, an extract of the song is attached. This shows the parts in the composition where transposition appears in the keys as it is played on. Below is the extract of the song:
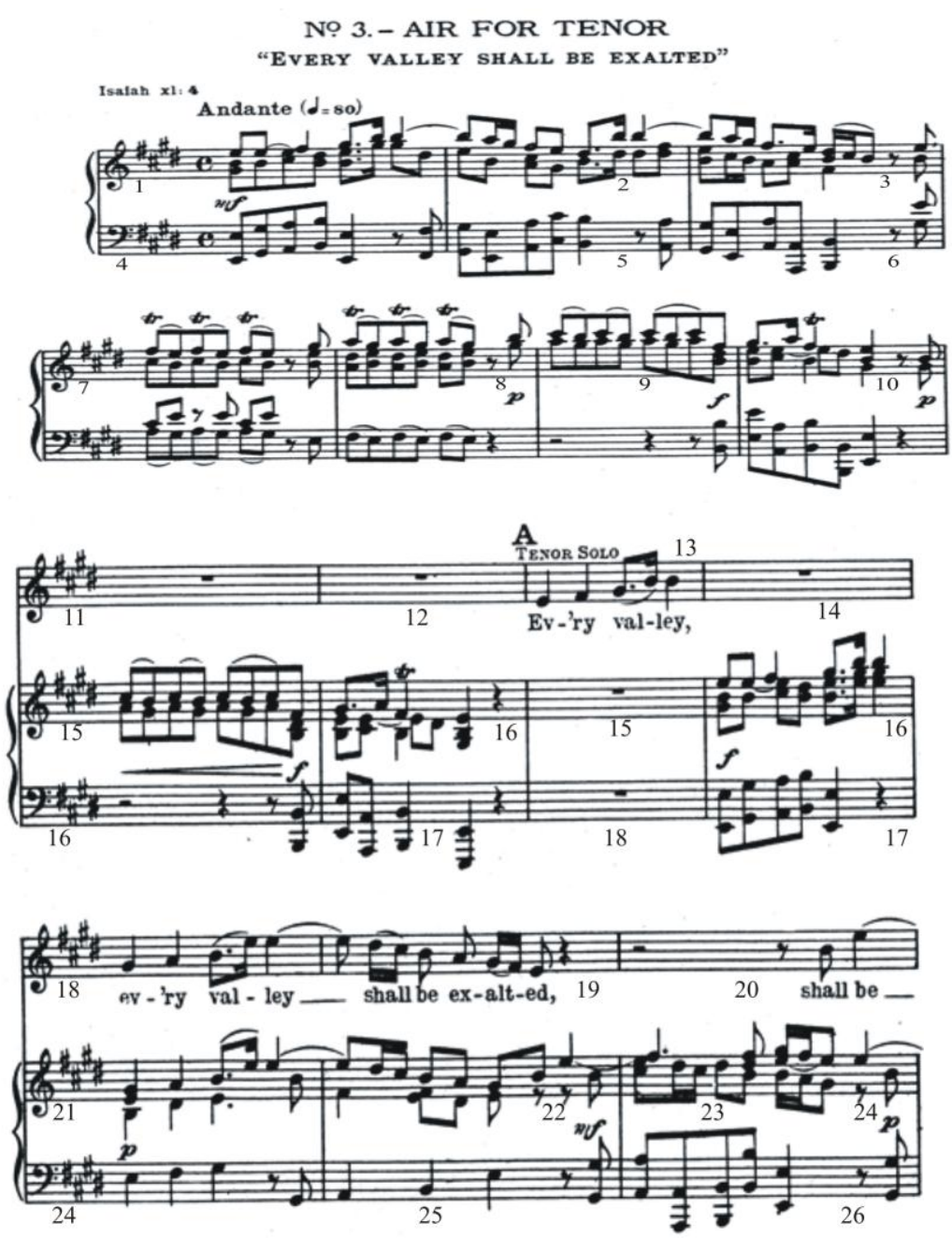

We can see that from bar 13 to 26 , the letter $A$ appeared, meaning the song is played from key of $A$. The song is then transposed to key of $B$, beginning from the $54^{\text {th }}$ bar, as shown below.

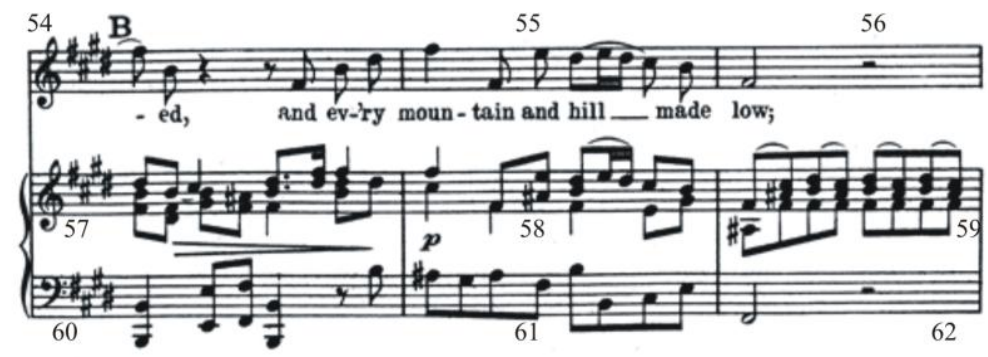

That is transposing from key of A to B. We show how this is made possible by application of the transposition formula. The diatonic scale of $A$ is given as; 


$$
A=\left\{A, B, C^{\#}, D, E F^{\#}, A^{b}\right\}=\quad\{d, r, m, f, s, l, t\}
$$

Transposing to $\mathrm{B}$, we have, given $T_{n}(x)=x+n=a \bmod 12$.

Let $\mathrm{n}=2$.

$x=\{9,1,4,8\}$, the $d$-chord of key of $A$;

$d$-chord of $B=\{11,3,6,10\}=\{d$ m st $\}$;

for $x=\{11,2,6,9\}$, the $r$-chord of key of $A$;

$r$-chord of $B=\{1,4,8,11\}=\{r f \mid d\}$;

for $x=\{1,4,8,11\}$, the $m$-chord of key of $A$;

$m$-chord of $B=\{3,6,10,1\}=\{m$ s t r $\}$;

for $x=\{2,6,9,1\}$, the $f$-chord of key of $A$;

f-chord of $B \quad=\{1,10,8,11\}=\{f \mid d m\}$;

for $x=\{4,8,11,2 \quad\}$, the s-chord of key of $A$;

$s$-chord of $B=\{6,10,1,4\}=\{s$ t r f $\}$;

for $x=\{6,9,1,4\}$, the I-chord of key of $A$;

I-chord of $B \quad=\{8,11,3,6\}=\{\mid d m s\}$;

for $x=\{8,11,2,6 \quad\}$, the $t$-chord of key of $A$;

$\mathrm{t}$-chord of $B \quad=\{10,1,4,8\}=\{t \quad r f \mid\}$.

We see also that from bar 110 , it is transposed to key of $C$.

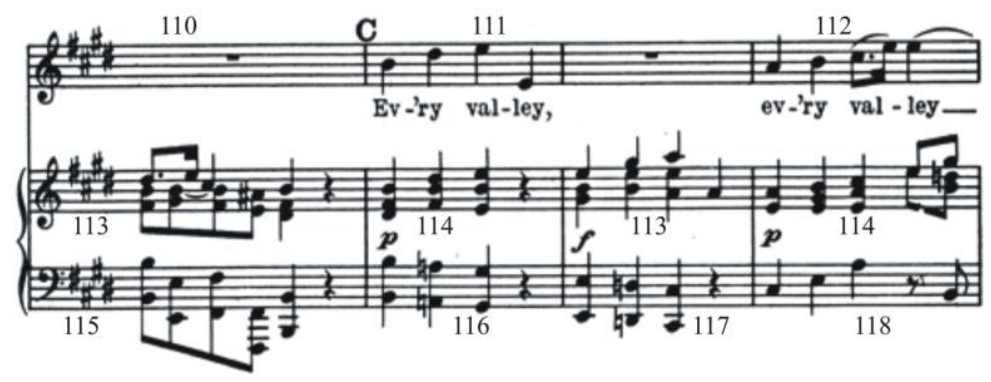

Explaining the transposition to key of $C$, we have:

$C=\{C, D, E, F, G, A, B\}=\{d, r, m, f, s, l, t\}$

Given $T_{n}(x)=x+n=a \bmod 12$

Let $\mathrm{n}=1$

$x=\{11,3,6,10\}, \quad$ the $d$-chord of key of $B$; 
$d$-chord of $C=\{0,4,7,11\}=\{d$ m s t $\}$;

for $x=\{1,4,8,11\}$, the $r$-chord of key of $B$;

$r$-chord of $A=\{2,5,9,0\}=\{r f \mid d\}$;

for $x=\{3,6,10,1\}$, the $m$-chord of key of $B$;

m-chord of $C=\{4,7,11,2\}=\{m$ s t r $\}$;

for $x=\{4,8,11,3\}$, the $f$-chord of key of $B$;

$f$-chord of $C=\{5,9,0,4\}=\{f \mid d m\}$;

for $x=\{6,10,1,4\}$, the s-chord of key of $B$;

$\mathrm{s}$-chord of $\mathrm{C}=\{7,11,2,5\}=\{\mathrm{s}$ t r f $\}$;

for $x=\{8,11,3,6\}$, the I-chord of key of $B$;

I-chord of $C=\{9,0,4,7\}=\{1 \mathrm{~d} \mathrm{~m} \mathrm{~s}\}$;

for $x=\{10,1,4,8\}$, the $t$-chord of key of $B$;

$\mathrm{t}$-chord of $\mathrm{C}=\{11,2,5,9\}=\{\mathrm{trf} \mid\}$.

Transposition of the form as in converting a song given in a key to another key is also applied in so many contemporary music, compose in scales major, while inversion is mostly seen in fugue music composition.

\section{MAIN RESULT}

Single line representation using images of elements in symmetric group is applied in this work.

\subsection{SONG COMPOSITION ON SYMMETRIC GROUP}

Collection of permutation group is known as symmetric group. The songs below are composed from the symmetric group of orders 4 and 5 .

For clarity, we state therefore that, the solfa notes will be represented in their respective numbers, since we are dealing with just diatonic scale and not chromatic scale, the number for each note regardless the key is noted as follows;

$\begin{array}{lllllll}\mathrm{d} & \mathrm{r} & \mathrm{m} & \mathrm{f} & \mathrm{s} & \mathrm{l} & \mathrm{t} \\ 1 & 2 & 3 & 4 & 5 & 6 & 7\end{array}$

We shall see how effective this will be in the composition of song in order 4 permutation group.

We have this song titled "Come Together" composed in Key of C major of order 4 permutation group. 


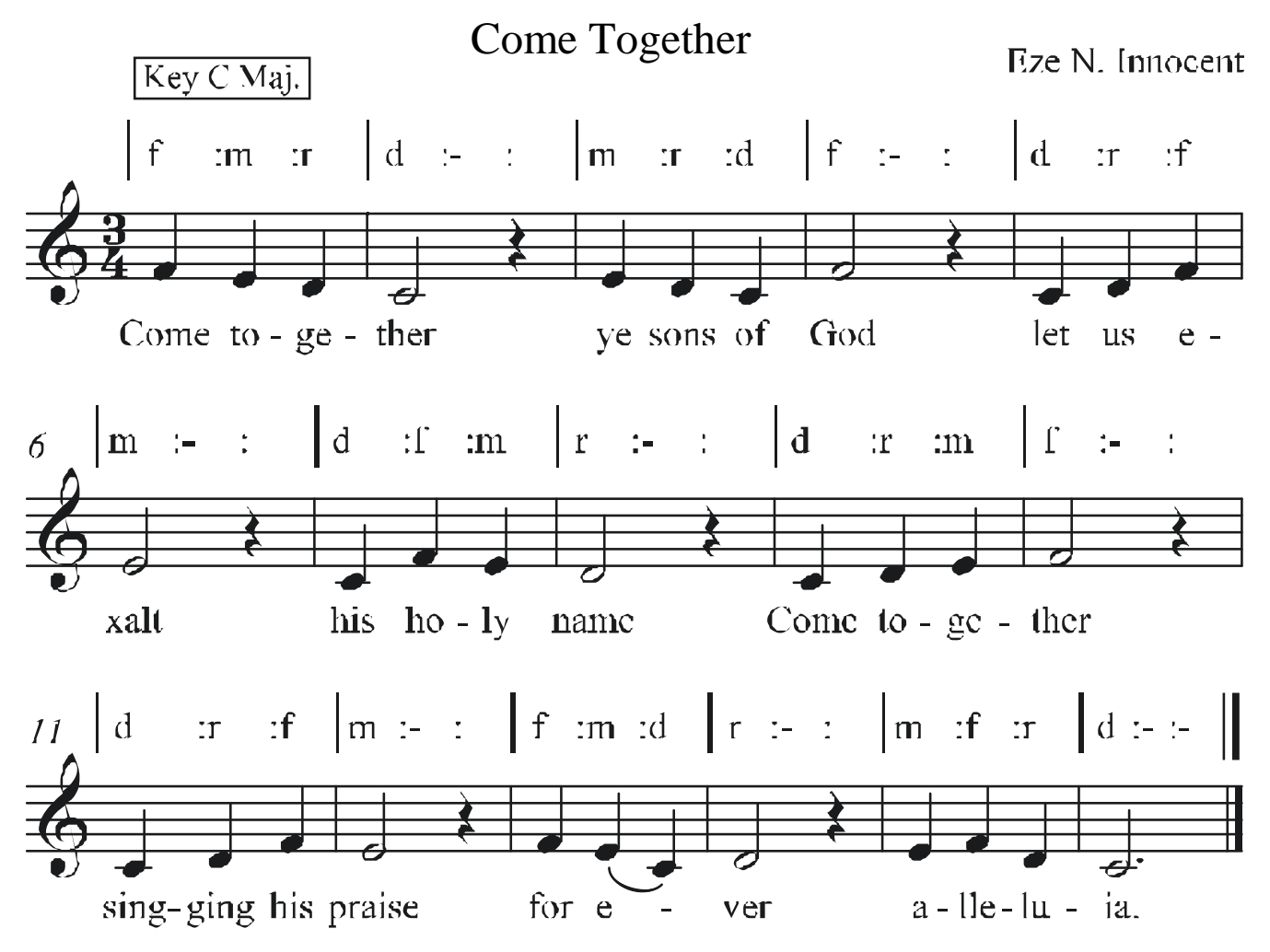

Writing out the solfa note in the song from the first bar to last bar, it will be discovered that this shows its composition in order 4 permutation group.

We have, using single line notation referring to the image:

$$
\begin{array}{ll}
\mathrm{fmrd}=4321=\left(\begin{array}{lll}
1 & 2 & 34 \\
4 & 3 & 21
\end{array}\right), & \mathrm{mrdf}=3214=\left(\begin{array}{lll}
1 & 2 & 34 \\
4 & 3 & 21
\end{array}\right) \\
\mathrm{drfm}=1243=\left(\begin{array}{lll}
1 & 2 & 34 \\
1 & 2 & 43
\end{array}\right), & \mathrm{d} \mathrm{fm}=1432=\left(\begin{array}{lll}
1 & 2 & 34 \\
1 & 4 & 32
\end{array}\right) \\
\mathrm{drmf}=1234=\left(\begin{array}{lll}
1 & 2 & 34 \\
1 & 2 & 34
\end{array}\right), & \mathrm{drfm}=1243=\left(\begin{array}{lll}
1 & 2 & 34 \\
1 & 2 & 43
\end{array}\right) \\
\mathrm{fm} \mathrm{dr}=4312=\left(\begin{array}{lll}
1 & 2 & 34 \\
4 & 3 & 12
\end{array}\right), & \mathrm{mfrd}=3421=\left(\begin{array}{lll}
1 & 2 & 34 \\
3 & 4 & 21
\end{array}\right)
\end{array}
$$

These are the members of elements used in the composition as seen in the script above.

Here we have another song composed in permutation group of order 5 . 


\section{Give Him All The Praise}

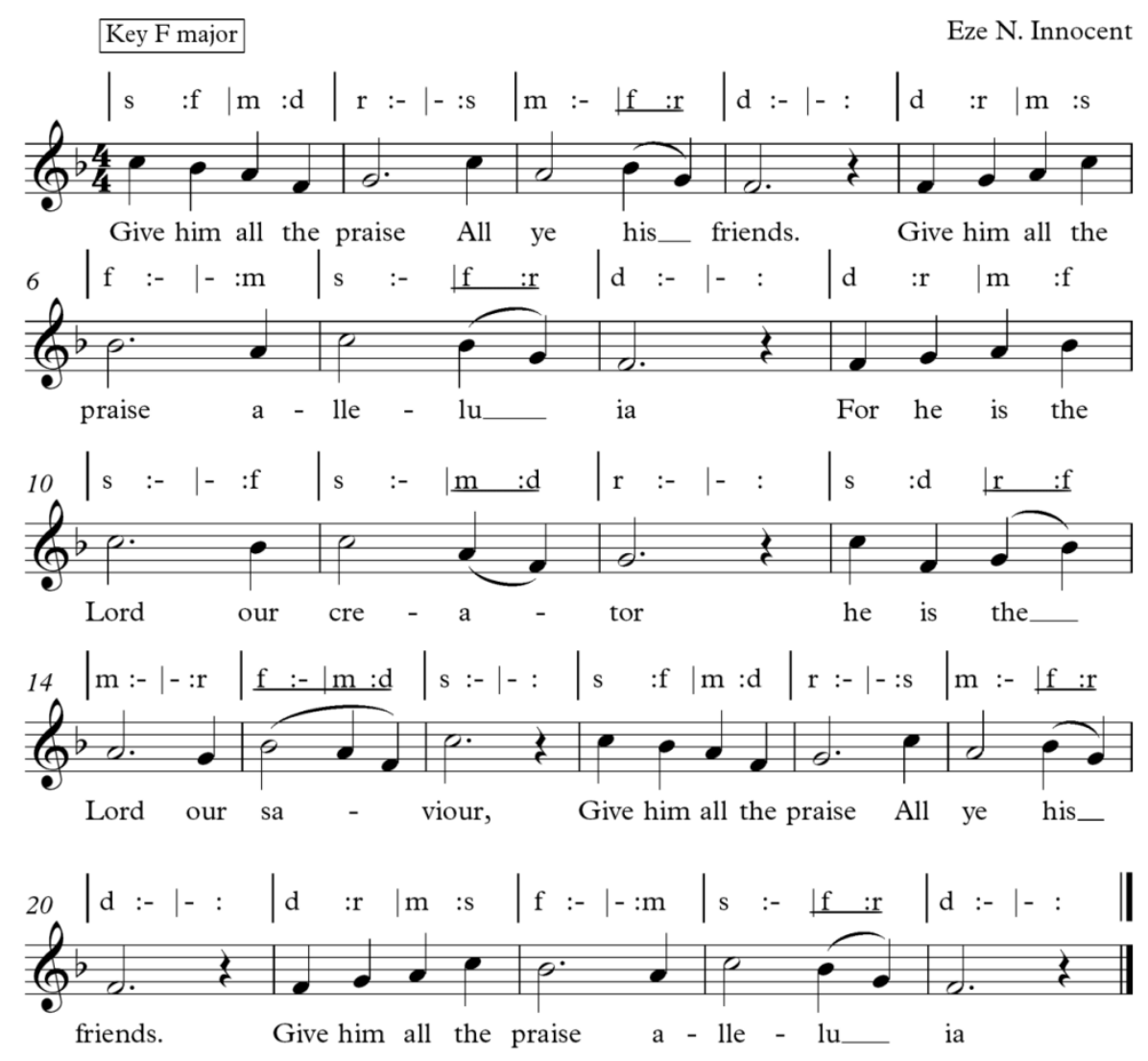

Showing the solfa notation of the song and relating it with the permutation group order 5 , starting from the first bar or measure, we have

$$
\begin{aligned}
& \mathrm{s} \mathrm{fmdr}=54321=\left(\begin{array}{llll}
1 & 2 & 34 & 5 \\
5 & 4 & 32 & 1
\end{array}\right), \\
& \operatorname{smfrd}=53421=\left(\begin{array}{llll}
1 & 2 & 34 & 5 \\
5 & 3 & 42 & 1
\end{array}\right) \text {, } \\
& \mathrm{drmsf}=12354=\left(\begin{array}{llll}
1 & 2 & 34 & 5 \\
1 & 2 & 35 & 4
\end{array}\right) \\
& \mathrm{msfrd}=35421=\left(\begin{array}{llll}
1 & 2 & 34 & 5 \\
3 & 5 & 42 & 1
\end{array}\right) \\
& \mathrm{drmfs}=12345=\left(\begin{array}{llll}
1 & 2 & 34 & 5 \\
1 & 2 & 34 & 5
\end{array}\right) \\
& \text { f s m d r }=45132=\left(\begin{array}{llll}
1 & 2 & 34 & 5 \\
4 & 5 & 13 & 2
\end{array}\right) \\
& \mathrm{sdrfm}=51243=\left(\begin{array}{llll}
1 & 2 & 34 & 5 \\
4 & 3 & 12 & 1
\end{array}\right) \\
& \mathrm{rfmds}=24315=\left(\begin{array}{llll}
1 & 2 & 34 & 5 \\
2 & 4 & 31 & 5
\end{array}\right)
\end{aligned}
$$


All the elements used in the composition are picked at random for convenience from among the elements of permutation group of orders 4 and 5 .

\section{Theorem 11.1}

A note of duration $d$ followed by $m$ dots has duration

$d_{m}=\frac{2^{m+1}-1}{2^{n+m}}$.

\section{Proof}

Since a note can be expressed as exponent, $\mathrm{n}$ of 2 , then for the duration of $\mathrm{m}$ given dotted note, the numerator is known combinatorically as

$\sum_{k=1}^{n}\left(\begin{array}{l}n \\ k\end{array}\right), n \geq 1$, which when compared is $2^{m+1}-1$ and $\mathrm{m}$ is noted to be an exponent. The duration is $\frac{2^{m+!}-1}{2^{n+m}}-$ ths of a beat

Hence, this result is equivalent to the one obtained in [4] as in section 8.

\section{CONCLUSION}

There is no limit to applications of algebra in music and one of such, is the composition of songs using random picking of elements from Symmetric group of order 4 and 5.

\section{REFERENCES}

1. Clough, John. (1979): "Aspects of Diatonic Sets." Journal of Music Theory 23/1 45-61.

2. Clough, John.(1994) "Diatonic Interval Cycles and Hierarchical Structure." Perspectives of New Music 32/1: 228-253.

3. Dave Benson (2007). Music: A Mathematical Offering. Cambridge University Press, Cambridge.

4. David Wright (2009), Mathematics and MusicbyCambridge University Press.

5. Laura Smoyer (2005), Musical Mathematics, (The Mathematical Structure of the Pythagorean and Equal Tempered Scale) by Fibson Publisher Corporation.

6. Steven A. Bleiler and Ewan Kummel (2016), Scales and Temperament from the Mathematical Viewpoint, Bridges Finland Conference Proceedings, 571 - 574.

7. Thomas M. Fiore (2011),Music and Mathematics, by Lead Publisher, London.

8. Timothy A. Johnson (2008), Foundations of Diatonic Theory: A Mathematically Based Approach to Music Fundamentals, by. Lanham, Md: Scarecrow Press. (Originally Published Emeryville, Ca: Key College Publishing, 2003.) 